\title{
Design of steering system of a buggy
}

\author{
D. Santana Sanchez, and A. Mostafa*
}

School of Environment, Science and Engineering, Southern Cross University, Lismore NSW 2480, Australia.

\begin{abstract}
The present paper discusses the design analysis and limitations of the steering system of a buggy. Many geometrical and performance characteristics of the designed steering system were considered to address the kinematic constraints and load carrying capacity of the steering elements. Ackremann geometry approach was used to assess the limiting steering angle, while Lewis bending formula with the inclusion of dynamic effects was employed to characterise the flexural properties of the rack and pinion steering system. Analytical results were numerically verified using ABAQUS/Explicit nonlinear finite element (FE) package. Good agreement has been achieved between analytical and numerical results in predicting the flexural behaviour of the steering rack and pinion system.
\end{abstract}

\section{Introduction}

The steering system plays a very important role throughout the entire design of a vehicle, as it is the interface between the driver and vehicle. As torque is provided by the driver onto the steering wheel, it rotates the steering column and entire steering mechanism as well. This leads to turning of the rotational motion into linear that transmitted to the wheels via the connected tie rods. Apart from having this function, steering systems allow for driver effort to be lowered in order to facilitate steering and make it more efficient. The components that make up the steering system also absorb a fraction of the road shock before making contact with the driver [1]. As several components make up a steering system, the complexity or simplicity are dependent on the desired features of the automobile and its geometrical limitations. It is noteworthy that the intended goal of the designed steering system is to ensure ease of use, effectiveness of operation and reliability of the mechanical components that last longer with minimal maintenance. Therefore, the introduction of general rack and pinion design process with dynamics factors according to the recommendations of the American Gear Manufacturers (AGMA) provides reliable analytical results, while FEA was required to build insightful picture of the stress and strain distributions across the areas of interest. The design of the steering shaft was performed following the distortion energy theory to determine the precise size based on static and fatigue stress concentrations. In general, the steering system of a vehicle consists of tierod linkages and steering arms forming a trapezoidal shape. As the system moves linearly, it forms an approximation of a parallelogram which skews one side according to the desired steering angle [2]. Ackermann principle for steering geometry is introduced in the current system to differentiate between the faster displacement rate of the outer wheel compared with the inner one. Front wheel steering and constant speed are some noteworthy hypotheses that are included in Ackermann principle which may affect the study of the slippage between the outer and inner wheels [3]. These aforementioned procedures were followed in order to achieve a comprehensive evaluation of a simplified steering system for the proposed buggy, as to study its mechanical performance under static loading.

\section{Design procedure of steering system}

\subsection{Ackermann percentage}

Ackermann percentage can be calculated according to the position of the steering arms (knuckles or Pitmans arms). This is done with the use of Freudenstein's equation [4], which is based on the known angles of the steering arms as the desired turn radius is set at $3.5 \mathrm{~m}$.

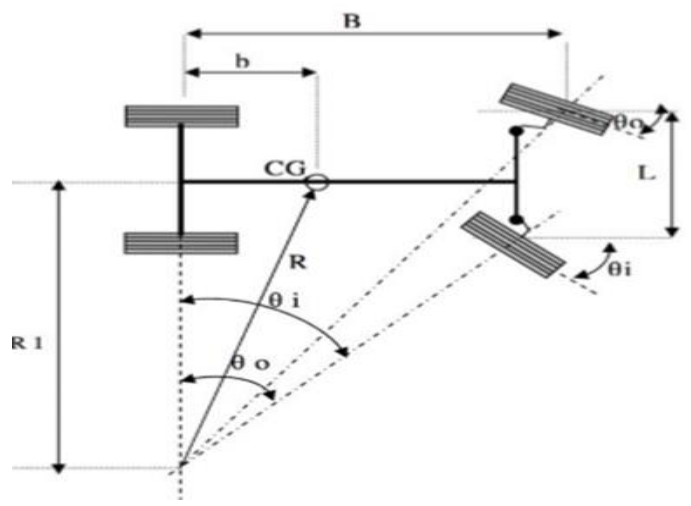

*Corresponding author: ahmed.thabet@scu.edu.au 


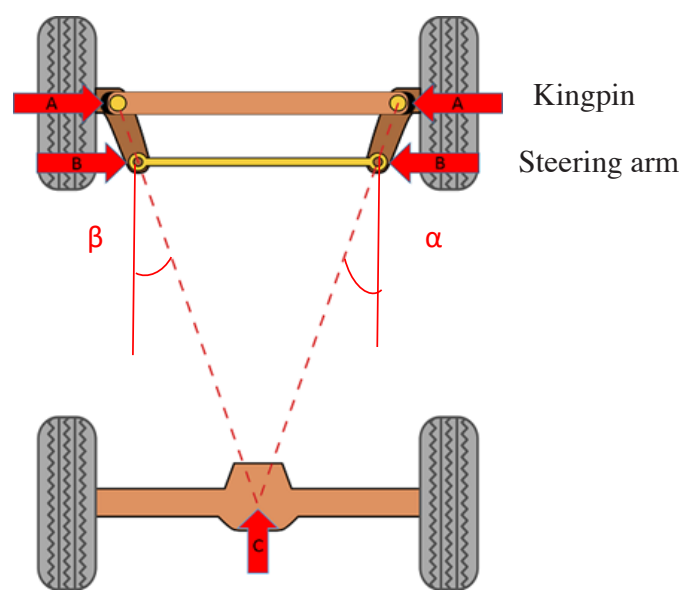

Fig. 1. Ackermann principle of steering angle [1].

Fig. 1 demonstrates the neutral position of the steering geometry, along with the kingpins and steering arms location. It can be seen that Ackermann's angle $\propto$ can be determined as:

$$
\propto=\tan ^{-1}\left(\frac{\left(\frac{S f}{2}\right)}{L}\right)
$$

Where $S f$ is the distance between the kingpin and the centre of the tyre and $L$ is the track-width.

\subsection{Steering geometry}

Fig. 1 shows the steering geometry required based on pro-Ackermann. The angles that each tyre is required can be determined based on the steer angle, hence rack travel can be determined.

$$
\begin{gathered}
R_{1}=\sqrt{R^{2}-B^{2}} \\
\theta_{i}=\tan ^{-1}\left(\frac{B}{R_{1}-\frac{L}{2}}\right) \\
\theta_{o}=\tan ^{-1}\left(\frac{B}{R_{1}+\frac{L}{2}}\right)
\end{gathered}
$$

Where $\theta_{o}, \theta_{i}, B, L, b, R_{1}$ and $R$ are the outside wheel turn angle, inside wheel turn angle, track width, wheel base, distance from rear axle to centre of mass, instantaneous centre of curvature and radius of turn, respectively. Therefore, the use of the greatest angle can be used in order to determine the steering ratio $(S . R)$, as per the desired steering effort of $270^{\circ} ; \quad(S . R=$ $270 / 41.38=6.52)$. Thus, for one complete turn of 160 mm steering wheel, the $S . R$ can be calculated using the following equation.

$$
S \cdot R=\frac{C}{(R . T)}
$$

Where S.R is the steering ratio based on desired steering effort and maximum angle needed to achieve a $3.5 \mathrm{~m}$ radius of turn, $C$ is the circumference of the steering wheel and R.T stands for rack travel.

In order to achieve centre to lock in both left and right directions, the calculated R.T should be doubled to accommodate for rack travel in both sides.

\subsection{Steering arm geometry}

The distance from the centre of the steering wheel to the pinion (Fig. 2) was chosen as $0.1 \mathrm{~m}$ to fit with the rest of the buggy's components, as it would be best suitable for the allocated space for the driver.
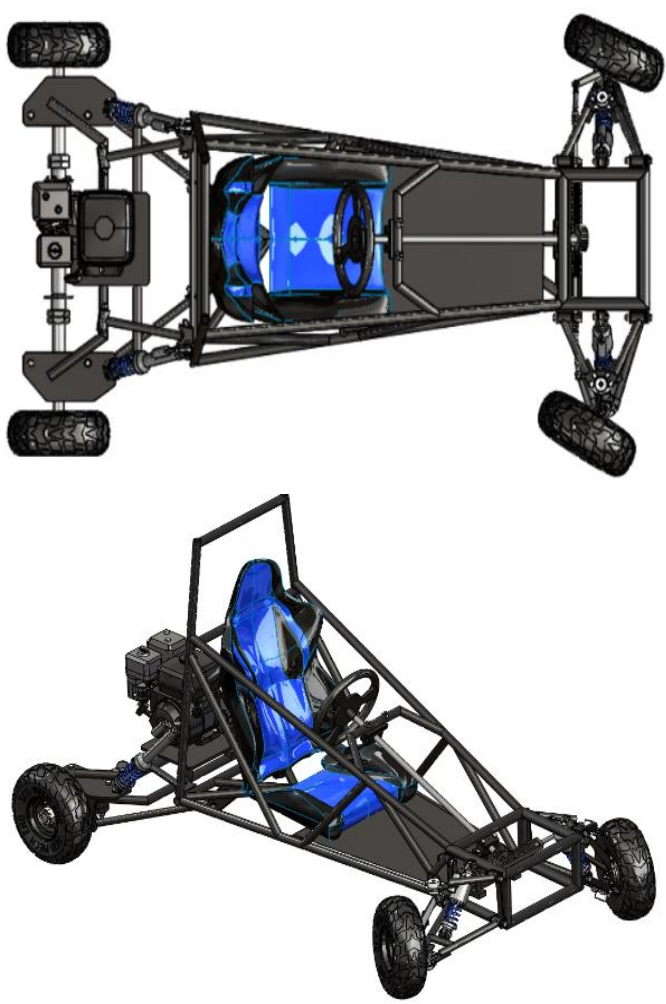

Fig. 2. Geometry of the designed buggy.

The minimum distance between the rack and the king pin can be calculated from the geometry of the steering arm (Fig. 3) as per the following equations:
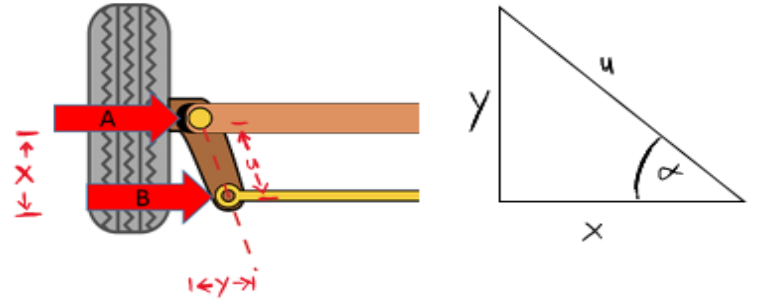

Fig. 3. Steering arm geometry.

$$
u=\frac{x}{\cos (\propto)}
$$




$$
\sin (\propto)=\frac{y}{u}
$$

Where $u, y, \mathrm{x}$ and $\propto$ are the steering arm length, arm base, distance away from king pin and Ackermann angle, respectively. The length of the overall tie rod can be determined by subtracting the arm base from the distance between the kingpins.

$$
\text { Lrod }=S f-2 y
$$

This distance must include the rack and tie rods. By employing the sum of moments equation and taking into account the static frictional force within a tyre, the force needed to turn the tyre at its greatest effort can be determined (in the current design, a lateral force of 201.354 $\mathrm{N}$ is needed).

\section{Analytical and numerical modelling of steering components}

\subsection{Analytical model of the rack and pinion}

The spur gear analysis was performed based on the amount of steering desired and the force that the gear is intended to transmit. Hence determining the pitch diameter $(D p)$ of the gear can be calculated based on the known requirements, as the gear is required to travel the rack from centre to lock in within $270^{\circ}$.

$$
D p=\frac{\left(2 * R . T * \frac{3}{4}\right)}{\pi}
$$

Followed by the number of teeth being determined:

$$
N=\frac{D p}{m}
$$

Where $N$ and $m$ are the number of teeth and module of the gear, respectively. Hence torque can be found as:

$$
T=F L * r p
$$

Where $F L$ and $r p$ are the lateral force and the radius of the pinion, respectively. This allows for the force at the steering wheel to be determined,

$$
T=F s t * r s t
$$

Where Fst and rst are the lateral force at steering wheel and the radius of the steering wheel, respectively. The dimensions of the rack was based on the dimensions of the pinion, thus a $3 \mathrm{~mm}$ module was adopted in order to minimize the number of teeth yet increasing the diameter of the pinion which lowered the lateral force. By utilizing the AGMA stress equation $(\sigma)$ and the Lewis bending equation [5], various dynamic factors can be considered and compared with the numerical results to determine the reliability and accuracy of the design. AGMA stress formula is given as:

$$
\sigma=\mathrm{W}^{\mathrm{T}} \mathrm{K}_{\mathrm{o}} \mathrm{K}_{\mathrm{v}} \mathrm{K}_{\mathrm{s}}\left(\frac{1}{\mathrm{bm}_{\mathrm{t}}}\right)\left(\frac{\mathrm{K}_{\mathrm{H}} \mathrm{K}_{\mathrm{B}}}{\mathrm{Y}_{\mathrm{J}}}\right)
$$

Where $W^{T}, K_{o}, K_{v}, K_{s}, b, m_{t}, K_{H}, K_{B}$ and $Y_{J}$ are the tangential transmitted load $(\mathrm{N})$, the overload factor, the dynamic factor, size correlation factor, the face width of the narrower member, transverse metric module, loaddistribution factor, rim-thickness factor and geometry factor for bending, respectively [3]. This would later allow for factors of safety of wear and bending to be concluded. In order to estimate the flexural strength of the pinion's teeth, Lewis bending formula $\left(\sigma_{L B}\right)$ was used (Equation 15). The formula allows for the determination of the teeth bending strength taking into consideration the dynamic characteristics of the applied load.

$$
\sigma_{L B}=\frac{K_{v} W}{F m Y}
$$

Where $K_{v}, W, F, m$ and $Y$ are the dynamic factor, tangential load $(\mathrm{N})$, face width, module and bending factor, respectively.

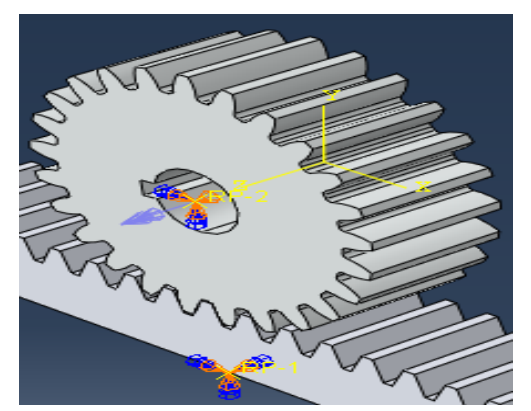

(a)

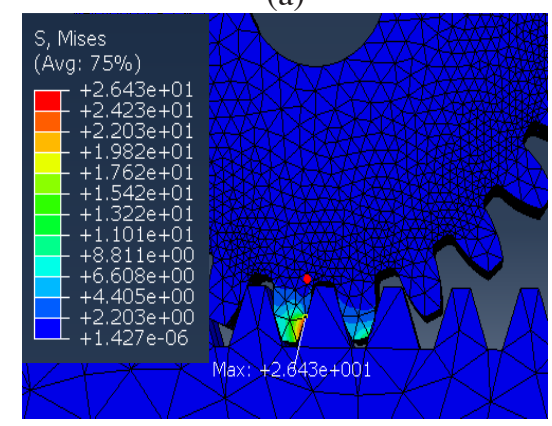

(b)

Fig. 4. Numerical modelling of the rack and pinion (a) boundary conditions and (b) Von Mises stress distribution.

\subsection{Numerical Model of the Rack and Pinion}

FE models were generated using ABAQUS software [6] to simulate the loading cases between the rack and pinion of the steering system. Both parts were modelled as linear elastic material with elastic modulus and Poisson's ratio equal $205 \mathrm{GPa}$ and 0.29 , respectively. 3D continuum element C3D10 was used to represent the geometry of the simulated parts to capture the stress concentration at the mating surfaces. Penalty contact interaction with 0.1 friction coefficient was used to model the tangential contact behaviour, while hard contact was utilized to prevent any pressure-overclosure 
at the interface. Based on the geometry, a total of 92209 quadratic tetrahedral elements were used with bias mesh distribution in the vicinity of the tooth face to capture the stress concentration in the pinion. The pinion was constrained from moving in all directions except the rotation around the $\mathrm{z}$-axis, while the rack was absolutely fixed to simulate the ultimate resisting load as shown in Fig. 4 (a).

Fig. 4 (b) shows the numerical distribution of the Von Mises stress with a maximum value of $26.43 \mathrm{MPa}$ at the tooth's face. The calculated flexural strength based on Lewis formula was $25.77 \mathrm{MPa}$, provided the average directional stress not the effective stress (Von Mises). This difference between the flexural strength based on Lewis formula and the effective stress could be attributed to the assumptions made in deriving Lewis equation such as the gear teeth are cantilever beam with load applied at the end of the tooth, radial forces are neglected and various factors such as sliding friction and stress concentrations based on changes of the area are neglected [5].

\section{Conclusions}

Analytical and numerical approaches were used to determine the reliability and the design constraints of the steering system of a buggy. Steering angle, ratio and geometry were estimated based on Ackermann principle to evaluate the kinematic constraints of the designed buggy. FE results based on static loading conditions have shown safe working conditions under selected design parameters. The employed rack and pinion have shown remarkable resistance to the applied bending load which ensures sufficient safety factor. Moreover, the analytical analysis using AGMA equation upon both rack and pinion provided results in close agreement with the FE ones.

\section{References}

1. A.; WJ, J. WG. Steering System Principles. Popular Mechanics; 2014.

2. Qiu Q, Fan Z, Meng Z, Zhang Q, Cong Y, Li B, et al. Extended Ackerman Steering Principle for the coordinated movement control of a four wheel drive agricultural mobile robot. Computers and Electronics in Agriculture. 2018;152:40-50.

3. Budynas RG, Nisbett JK, Shigley JE. Shigley's mechanical engineering design2015.

4. Ghosal A. The Freudenstein Equation. Design of Fourl-Link Mechanisms. 2010.

5. Budynas RG, Nisbett JK. Mechanical Engineering Design. New York: Mc Graw HIll Education; 2015.

6. ABAQUS. ABAQUS 2018 EF Documentation. 2018 ed: Simulia; 2018. p. Finite Element Analysis. 